\title{
A New Era in the Adjuvant Treatment of Pancreatic Adenocarcinoma
}

\author{
Manuela Machado $^{1}$, Maria Leitão ${ }^{1}$, Mário Fontes e Sousa ${ }^{2}$, Magalhães e Helena ${ }^{3}$ \\ ${ }^{1}$ Oncologia Médica, IPO Porto, Porto, Portugal \\ ${ }^{2}$ Oncologia Médica, Centro Hospitalar Lisboa Ocidental (CHLO), Lisboa, Portugal \\ ${ }^{3}$ Oncologia Médica, Unidade Local de Saúde de Matosinhos, Hospital Pedro Hispano, Matosinhos, Portugal \\ Email: m.machado.fn@gmail.com
}

How to cite this paper: Machado, M., Leitão, M., e Sousa, M.F. and e Helena, M. (2019) A New Era in the Adjuvant Treatment of Pancreatic Adenocarcinoma. Journal of Cancer Therapy, 10, 915-919.

https://doi.org/10.4236/jct.2019.1012078

Received: October 14, 2019

Accepted: December 7, 2019

Published: December 10, 2019

Copyright (c) 2019 by author(s) and Scientific Research Publishing Inc. This work is licensed under the Creative Commons Attribution International License (CC BY 4.0).

http://creativecommons.org/licenses/by/4.0/

\begin{abstract}
Pancreatic adenocarcinoma is currently a major public health problem, being the 7th cause of death worldwide. Incidence is increasing and unfortunately nowadays the incidence is almost overlapping to mortality. The cornerstone of curative treatment is still surgery, but adjuvant treatment is critical to decreasing the risk of recurrence.
\end{abstract}

\section{Keywords}

Pancreatic Adenocarcinoma, Adjuvant Chemotherapy, Positive Clinical Trials

\section{Introduction}

Most malignant pancreatic tumors (95\%) arise from the exocrine pancreas and of these $85 \%$ are adenocarcinomas. Currently, the only treatment considered curative is surgical resection. Yet, at diagnosis, only $10 \%-20 \%$ of patients are suitable for surgery and even in these rare patients, the 5 -year survival is $<10 \%$; the recurrence rate at the 2 -year is $\sim 69 \%-75 \%$. Clearly, these high-risk patients warrant adjuvant treatment [1] [2] [3].

For over a decade, the standard adjuvant treatment has been chemotherapy (ChT), with Gemcitabine (GEM) monotherapy, 5-Fluorouracil (5-FU)/Leucovorin (LV), or in Asia, S1.

In recent years, after the results of phase III trials, ESPAC-4 and PRODIGE 24/CCTG PA.6, the standard for adjuvant chemotherapy became modified FOLFIRINOX (mFOLFIRINOX), or in some cases, GEM associated to Capecitabine (GEM/CAP). 


\section{Materials and Methods}

The authors have conducted a qualitative systematic review, using the PubMed search engine, of all phase III prospective randomized trials in the adjuvant setting using chemotherapy for pancreatic adenocarcinoma. The trial selection consisted of both statistically significant and clinically relevant works, from January 2007 to October 2019. Negative trials were excluded. We focused mainly on reports of overall survival, disease free survival, objective response rate and impact on quality of life.

\section{Article}

Among the worldwide causes of death, pancreatic adenocarcinoma ranks in 7th place, in both men and women and its incidence is increasing [1], possibly concerning an ageing population and lifestyle changes.

Surgical resection is the only potentially curative treatment; nevertheless only $15 \%-20 \%$ of the patients are suitable to surgery, and yet this small group experiences a very high recurrence rate $(>80 \%)$ and dismal prognosis, with 5 -year overall survival of only $9 \%-10 \%$, and 45,750 estimated deaths in 2019 [2] [3] [4] [5].

Such disappointing numbers lead to different strategies that should be discussed in a multidisciplinary scenario: adjuvant chemotherapy, adjuvant radiotherapy or both.

Here, the authors will highlight the positive randomized trials evaluating adjuvant chemotherapy.

\section{Results/Discussion}

The clinical trials RTOG 9704, CONKO-001, ESPAC 3 and JASPAC 1 demonstrated improvement in overall survival (OS) and in progression free survival (PFS) [6].

Up to 2016, the adjuvant standard treatment was 6 months of GEM in monotherapy, or 5FU/LV, or in Asian patients, S1. However, in these trials, even patients who received adjuvant chemotherapy, experienced 2 -year recurrence rate of $75 \%$.

More recently, two practice-changing trials established the new adjuvant treatment following resection of pancreatic cancer: ESPAC-4 and PRODIGE 24/CCTG PA.6.

In the CONKO-001 trial, 368 operated patients with $\mathrm{R} 0$ and $\mathrm{R} 1$ resections were randomized in 2 groups: ChT with GEM $\left(1000 \mathrm{mg} / \mathrm{m}^{2}\right.$, in a $30 \mathrm{~min} \mathrm{infu-}$ sion, days 1,8 and 15 every 38 days, 6 cycles; $\mathrm{n}=179)$ vs observation $(\mathrm{n}=175)$. The primary objective of the trial was DFS, which was clearly superior in the treatment arm (13.4 vs $6.7 \mathrm{~m}$; Hazard ratio (HR) 0.55; p < 0.001). The same was observed in the OS, which was 22.8 months in the GEM group vs 20.2 months (HR 0.76; $\mathrm{p}=0.01$ ) [7] [8].

In another trial (ESPAC 3), 1088 patients with pancreatic adenocarcinoma 
were randomized after surgery ( $\mathrm{R} 0$ and $\mathrm{R} 1$ resections) in 2 adjuvant ChT groups: GEM $\left(1000 \mathrm{mg} / \mathrm{m}^{2}\right.$, in a $30 \mathrm{~min}$ infusion, days 1,8 and 15 every 38 days, 6 cycles, $\mathrm{n}=537)$ vs $\mathrm{AF}\left(20 \mathrm{mg} / \mathrm{m}^{2}\right.$ day 1$)$ and $5-\mathrm{FU}\left(425 \mathrm{mg} / \mathrm{m}^{2}\right.$ in bolus, days 1 and 5$)$ every 28 days, 6 cycles. OS was similar in both groups (23.6 vs 23 months; HR 0.94; $\mathrm{p}=0.39)$ and so was the DFS (14.3 vs 14.1 months, HR 0.55, $\mathrm{P}<0.001)$ [9].

In the non-inferiority phase III trial JASPAC 01 , which included 385 operated patients with pancreatic adenocarcinoma, with R0 and R1 surgeries in 33 hospitals in Japan, the randomization was carried out in 2 groups of adjuvant ChT: S-1 $(80 / 100 / 120 \mathrm{mg} /$ day according to BSA per os twice a day, days 1 to 28 , every 42 days, 4 cycles, $\mathrm{n}=192)$ and GEM $\left(1000 \mathrm{mg} / \mathrm{m}^{2}\right.$, in a $30 \mathrm{~min}$ infusion, days 1,8 and 15 every 38 days, 6 cycles, $n=193$ ). The relapse free survival was 22.9 months in the S-1 group vs 11.3 months in the GEM group (HR: $0.60 ; p<0.0001$ ), with a 5 -year DFS of 33.3 vs $16.8 \%$. OS was also superior in patients treated with S-1 (46.5 vs 25.5 months; HR: 0.57 ; p < 0.0001 ) with 5 -year OS of $44.1 \%$ vs $24.4 \%$. Some experts justified this advantage with S-1 with the arguments that all patients were from Asian ethnicity. There was a higher exposure (dose/intensity) in the S-1 treatment (28 consecutive days) and presumably a better absorption of S-1 after a pancreatic resection [10].

In recent years, two studies changed the standard of care in adjuvant treatment of pancreatic adenocarcinoma.

In 2016, the ESPAC 4 phase III trial was published. It randomized, in 1:1 way, 732 patients, after surgical resection (either R0 or R1) and performance status (PS) $\leq 2$, within 12 weeks after surgery, to two arms:

- GEM (1000 mg/m², 30 minutes infusion, days 1, 8 and 15) associated to CAP $\left(1660 \mathrm{mg} / \mathrm{m}^{2}\right.$ per day, twice daily, oral, for 21 days), in 28 days cycle, 6 cycles.

- GEM (1000 mg/m², 30 minutes infusion, days 1, 8 and 15), in 28 days cycle, 6 cycles.

The primary endpoint was OS, while PFS was a secondary endpoint. Toxicity and quality of life were evaluated. With a median 43.2 months follow-up, there was a survival benefit in the combination arm vs monotherapy (OS 28 months vs 25.5 months, respectively, with HR 0.82; $\mathrm{p}=0.032$ ). In the R0 subgroup, the difference was even striking: 39.5 months vs 27.9 months; on the other hand, in R1 resection, the survival was similar: 23.7 months vs 23 months. The PFS was similar (13.9 months vs 13.1 months; HR $0.86, \mathrm{P}=0.082$ ). Not surprisingly, toxicity was higher in the combination arm, but considered manageable. The study was criticized because there was no cut-off for CA 19.9 tumor marker, namely only $662 / 730$ patients $(90.7 \%)$ the value was actually known. Additionally, the baseline CT scan was allowed up to 12 weeks before randomization, the follow-up protocol was not standardized (variation between centers) and the population was considered very high risk for recurrence $(61 \% \mathrm{R} 1$ resections and $80 \% \mathrm{~N}+$ ) [11].

In the multicenter, randomized, phase 3 trial PRODIGE 24-ACCORD and CCTG PA 6, a mFOLFIRINOX regimen was compared to GEM among resected pancreatic cancer patients [12]. In this trial, 493 patients were included: 247 to 
mFOLFIRINOX (oxaliplatin $85 \mathrm{mg} / \mathrm{m}^{2}$, leucoverin $400 \mathrm{mg} / \mathrm{m}^{2}$, irinotecan 180 $\mathrm{mg} / \mathrm{m}^{2}$, and fluorouracil $2400 \mathrm{mg} / \mathrm{m}^{2}$ continuous infusion in $46 \mathrm{~h}$, every 14 days for 24 weeks) vs GEM $1000 \mathrm{mg} / \mathrm{m}^{2} \mathrm{D} 1,8,15$ every 28 days for 24 weeks. After a safety analysis, the dose of irinotecan was reduced to $150 \mathrm{mg} / \mathrm{m}^{2}$ and omission of fluorouracil bolus.

The primary endpoint was disease free survival (DFS), which was superior in the mFOLFIRINOX arm: 21.6 vs 12.8 months in GEM arm (HR 0.58; $\mathrm{p}<0.001$ ), and this benefit occurred across all groups, including with adverse prognostic characteristics, like positive lymph nodes, R1 surgery, and T3 or T4 tumours.

The overall survival (median: 54.4 vs 35 months, HR 0.64, p $<0.003$ ), metastasis free survival (median: 30.4 vs 17.7 months; HR 0.59, p > 0.001) and cancer-specific survival (not reached vs 36.4 months; HR 0.63, $\mathrm{p}=0.003$ ) were also improved in the mFOLFIRINOX group compared with GEM, however there were more adverse effects $\mathrm{G} 3$ or 4 in the mFOLFIRINOX arm (75.9\%) than in the GEM arm (52.9\%).

Regarding these impressive outcomes in efficacy, and the longest overall survival ever reported in a trial, we have completely changed our practice in many patients with resected pancreatic adenocarcinoma and good performance status.

\section{Conclusions}

The ESPAC-4 and PRODIGE 24/CCTG PA.6 led to changes in the standard of care in the adjuvant treatment in pancreatic adenocarcinoma. Currently, in patients successfully resected and with good performance status, the preferred regimen is mFOLFIRINOX. Meanwhile, in frail or older patients, particularly in R0 resections, the GEM/CAP (in some cases even GEM monotherapy) is an option to consider.

The treatment decision should always consider the specific patient, in particular the comorbidities and expectations.

\section{Conflicts of Interest}

The authors declare no conflicts of interest regarding the publication of this paper.

\section{References}

[1] Bray, F., Ferlay, J., Soerjomataram, I., Siegel, R.L., Torre, L.A. and Jemal, A. (2018) Global Cancer Statistics 2018: GLOBOCAN Estimates of Incidence and Mortality Worldwide for 36 Cancers in 185 Countries. CA: A Cancer Journal for Clinicians, 68, 394-424. https://doi.org/10.3322/caac.21492

[2] Riall, T.S. and Lillemoe, K.D. (2007) Underutilization of Surgical Resection in Patients with Localized Pancreatic Cancer. Annals of Surgery, 246, 181-182. https://doi.org/10.1097/SLA.0b013e31811eaa2c

[3] Hernandez, J.M. (2010) The Natural History of Resected Pancreatic Cancer without Adjuvant Chemotherapy. The American Journal of Surgery, 76, 480-485.

[4] Rawlaa, P., Sunkarab, T. and Gaduputi, V. (2019) Epidemiology of Pancreatic Cancer: Global Trends, Etiology and Risk Factors. World Journal of Oncology, 10, 
10-27. https://doi.org/10.14740/wjon1166

[5] SEER Database. https://seer.cancer.gov/statfacts/html/pancreas.html

[6] NCCN Guidelines Version 1.2020. Pancreatic Adenocarcinoma: Postoperative-Adjuvant Therapy, $41 \mathrm{p}$.

[7] Oettle, H., et al. (2013) Adjuvant Chemotherapy with Gemcitabine and Long-term Outcomes among Patients with Resected Pancreatic Cancer. The CONKO-001 Randomized Trial JAMA, 310, 1473-1481. https://doi.org/10.1001/jama.2013.279201

[8] Oettle, H., et al. (2007) Adjuvant Chemotherapy with Gemcitabine vs Observation in Patients Undergoing Curative-Intent Resection of Pancreatic Cancer: A Randomized Controlled Trial. JAMA, 297, 267-277. https://doi.org/10.1001/jama.297.3.267

[9] Neoptolemos, J.P., et al. (2010) Adjuvant Chemotherapy with Fluorouracil plus Folinic Acid vs Gemcitabine Following Pancreatic Cancer Resection: A Randomized Controlled Trial. JAMA, 304, 1073-1481. https://doi.org/10.1001/jama.2010.1275

[10] Uesaka, K., et al. (2016) Adjuvant Chemotherapy of S-1 versus Gemcitabine for Resected Pancreatic Cancer: A Phase 3, Open-Label, Randomized, Non-Inferiority Trial (JASPAC 01). Lancet, 388, 248-257.

https://doi.org/10.1016/S0140-6736(16)30583-9

[11] Neoptolemos, J.P, et al. (2017) Comparison of Adjuvant Gemcitabine and Capecitabine with Gemcitabine Monotherapy in Patients with Resected Pancreatic Cancer (ESPAC-4): A Multicentre, Open-Label, Randomised, Phase 3 Trial. Lancet, 389, 1011-1024. https://doi.org/10.1016/S0140-6736(16)32409-6

[12] Conroy, T., et al. (2018) FOLFIRINOX or Gemcitabine as Adjuvant Therapy for Pancreatic Cancer. The New England Journal of Medicine, 379, 2395-2406. https://doi.org/10.1056/NEJMoa1809775 